\title{
The most frequent Polish ATM mutations are not susceptibility factors for tobacco-related cancers
}

\author{
Marta Podralska ${ }^{1}$, Agnieszka Dzikiewicz-Krawczyk ${ }^{1}$, Maria Mosor ${ }^{1}$, Magdalena Żurawek ${ }^{1}$, \\ Katarzyna Iżykowska ${ }^{1}$, Ryszard Stomski ${ }^{1}$, Małgorzata Rydzanicz², Piotr Gabryel ${ }^{3}$, \\ Wojciech Dyszkiewicz ${ }^{3}$, Iwona Ziółkowska-Suchanek ${ }^{1}$
}

${ }^{1}$ Institute of Human Genetics, Polish Academy of Sciences, Poznan, Poland 2Department of Medical Genetics, Medical University of Warsaw, Warsaw, Poland ${ }^{3}$ Department of Thoracic Surgery, Poznan University of Medical Sciences, Poznan, Poland

Submitted: 28 May 2018; Accepted: 10 October 2018

Online publication: 8 April 2020

Arch Med Sci 2021; 17 (5): 1158-1163

DOI: https://doi.org/10.5114/aoms.2020.94155

Copyright @ 2020 Termedia \& Banach

\section{Abstract}

Introduction: The inactivation of both alleles of the ATM gene leads to ataxiatelangiectasia syndrome, whereas carriers of monoallelic mutations in the ATM gene are associated with increased risk of different types of cancer. Three substitutions in the ATM gene (c.6095G >A, c.7630-2A>C, c.5932G $>$ T) are the most common mutations causing ataxia-telangiectasia among Polish patients. The aim of this study was to determine whether these ATM mutations are associated with increased risk of tobacco-related cancers.

Material and methods: 783 Polish patients with tobacco-related cancers were included in the study (468 with lung cancer, 153 with a single laryngeal cancer, 86 with multiple primary tumors localized in the larynx and 76 multiple primary tumors localized in the head or neck). The control group consisted of 464 healthy subjects from the Polish population. Three ATM mutations - c.5932G >T, c.6095G >A, c.7630-2A>C - were tested among selected patients. Molecular analyses were performed using high resolution melting analysis and restriction fragment length polymorphism.

Results: In the present study, we detected only one mutation, c.7630-2A>C, and no carriers of c.5932G >T, c.6095G >A mutations in the ATM gene among Polish patients with tobacco-related cancers. A patient with c.7630-2A>C mutation was diagnosed with lung adenocarcinoma, the most common type of lung cancer. One carrier of c.6095G>A mutation was found in the control group.

Conclusions: The results indicate that the studied ATM variants do not seem to be associated with tobacco-related cancers in Poland.

Key words: ATM mutations, Polish population, tobacco-related cancers.

\section{Introduction}

Mutations of DNA repair genes may be associated with impaired carcinogen-induced DNA damage repair and thus may influence the individual risk of cancer. One of the genes involved in the DNA repair process is the ataxia-telangiectasia mutated gene (ATM). ATM kinase is involved in maintaining genome integrity and stability. The ATM protein plays an important role in the repair of DNA double-strand breaks and is involved in cell-cycle regulation and apoptosis. Homozygous or compound heterozygous mutations in the ATM gene are responsible for pro-
Corresponding author: Marta Podralska Institute of Human Genetics Polish Academy of Sciences 32 Strzeszyńska St 60-479 Poznan, Poland Phone: +48 616579207 E-mail: mpod@man.poznan.pl 
gressive neurodegenerative, autosomal recessive disorder - ataxia telangiectasia (AT). The risk of developing cancer in AT patients is higher compared to individuals in the general population. Among AT patients, the most common types of cancers are leukemia and lymphoma. Moreover, monoallelic mutations in the ATM gene are associated with increased risks of developing cancer. There is evidence for an increased risk of breast cancer among heterozygous ATM mutation carriers [1]. Both family-based and population-based approaches indicated that carriers of some ATM variants have moderate breast cancer risk. Prior studies also revealed an association between monoallelic mutations and increased risks of gastric cancer [2]. Moreover, it was also shown that mutations in the ATM gene predispose to hereditary pancreatic cancer $[3,4]$, whereas loss-of-function mutations were identified in patients with prostate cancer [5].

Head and neck cancers and lung cancers remain a major health problem worldwide and are caused by the interaction between genetic susceptibility and environmental influences. Alcohol consumption and tobacco use are the two most important risk factors for these types of cancer. Cigarette smoke activates ATM through its phosphorylation on Ser1981 and histone H2AX phosphorylation as a result of carcinogenic DNA double-strand break formation (DSB) [6].

In our previous study it was shown that the heterozygous p.I171V mutation of the NBN gene, involved in DSB repair, may contribute to laryngeal cancer $(O R=11.70)$ and multiple primary tumors $(O R=28.35)$ [7]. We observed a significantly increased risk of lung cancer for carriers of the p.I171V variant $(\mathrm{OR}=7.76)$ [8].

The current study aimed to determine whether the most frequent ATM mutations may predispose to tobacco-related cancers in Polish patients. Three ATM mutations - c.5932G >T, c.6095G >A, c.7630-2A $>C$ - were tested among 468 patients with lung cancer, 153 with a single laryngeal cancer, 86 with multiple primary tumors of head and neck, and 464 healthy individuals from the Polish population.

\section{Material and methods}

\section{Patients}

The cohort of patients with tobacco-related cancers was recruited in the Department of Thoracic Surgery of the University of Medical Sciences in Poznan (Poznan, Poland). All of the studied subjects were of Caucasian ancestry from the Great Poland region of Poland. Blood samples were collected from 783 Polish patients: 468 with lung cancer (LC), 153 with a single laryngeal cancer
(LyC) and 86 with multiple primary tumors but one malignancy (primary or second primary) localized in the larynx (MPT-LyC) and 76 multiple primary tumors (MPT) localized in the head or neck. Almost all cancer patients had positive history of tobacco smoking. The detailed characteristics of LC cases and MPT-LyC/MPT patients were presented in our previous studies [9-11]. Most of the LC patients were male $(69 \%)$, with median age of 63.5 years (range: $51-78$ years). Almost all LC cases were non-small cell lung cancer. Squamous cell carcinomas occurred in 208 patients (46\%), adenocarcinoma in 185 cases (41\%). None of the LyC patients with a single laryngeal cancer developed any second primary tumor during the 5-year observation period. All LyC cases were squamous cell carcinomas. All patients were adult men aged 41-81 years. The cohort of MPT-LyC/MPT patients fulfilled all the Warren and Gates criteria. In all MPT-LyC patients the index or the second tumor was laryngeal cancer, whereas in MPT patients the index or the second tumor was localized in the head and neck region, excluding the larynx.

\section{Controls}

Overall 464 anonymous blood samples were used as population controls. This group consisted of individuals for screening check-up in hospital or healthy blood donors with negative history of medical illnesses. Efforts were undertaken to frequency-match cases by ethnicity, age and sex. Most of them were male (72\%), with median age of 55 years (range: $35-80$ years).

The study was conducted with the approval of the Central Ethical Committee of the Ministry of Health, Poland, in accordance with the tenets of the Helsinki Declaration. All patients signed informed consent forms.

\section{Molecular analysis}

DNA was extracted from blood samples using the columns provided in the QIAamp DNA Blood Mini Kit (Qiagen GmbH, Hilden, Germany), according to the manufacturer's instructions.

ATM mutations (c.5932G $>$ T, c.7630-2A $>C$ ) in exons 42 and 54 were assessed by restriction fragment length polymorphism (RFLP) analysis of polymerase chain reaction (PCR) products, using Msel and Alul restriction enzymes (Thermo Fisher Scientific, Inc., Pittsburgh, PA, USA), respectively. The genomic DNA was amplified using a previously reported primer set, flanking exons 42 and 54 of the ATM gene. In both cases, PCR reactions were performed in a total volume of $25 \mu \mathrm{l}$ containing $2.5 \mu \mathrm{l}$ of $10 \mathrm{X}$ PCR buffer with $15 \mathrm{mM} \mathrm{MgCl}_{2}$ (Sigma-Aldrich, St. Louis, MO, USA), $5 \mathrm{pM}$ of each primer, $2 \mathrm{mM}$ of each 
deoxynucleotide triphosphate (dNTP; SigmaAldrich, Steinheim, Germany), 1 unit of Taq DNA polymerase (Sigma-Aldrich, St. Louis, MO, USA) and $25 \mathrm{ng}$ of DNA template. Amplification conditions involved initial denaturation for $300 \mathrm{~s}$ at $95^{\circ} \mathrm{C}$, followed by 35 cycles of $20 \mathrm{~s}$ at $95^{\circ} \mathrm{C}, 20 \mathrm{~s}$ at $57^{\circ} \mathrm{C}$ and $60 \mathrm{~s}$ at $72^{\circ} \mathrm{C}$, with a $600 \mathrm{~s}$ final extension at $72^{\circ} \mathrm{C}$. Next, $10 \mu \mathrm{l}$ of the PCR products were digested for $16 \mathrm{~h}$ at $37^{\circ} \mathrm{C}$, and analyzed by electrophoresis on a $2 \%$ agarose gel, in the presence of ethidium bromide.

The c.5932G>T mutation creates the Msel restriction site. After digestion of the 232 bp PCR product containing mutation, Msel endonuclease produces three restriction fragments, $159 \mathrm{bp}$, $40 \mathrm{bp}$ and $33 \mathrm{bp}$. The endonuclease Alul recognizes the AG $\downarrow C T$ sequence, cleaves the PCR fragments containing the mutation C.7630-2C and generates 213, 61, 7-bp fragments, whereas the wild-type PCR fragments are cleaved in three restriction sites and four bands are observed (143, 70, 61, 7-bp).

The third mutation, c.6095G $>A$, in exon 43 of the ATM gene, was analyzed using a high-resolution melting (HRM) assay. Each $20-\mu$ l reaction volume comprised $25 \mathrm{ng}$ of purified genomic DNA, $10 \mu \mathrm{l}$ of reaction mix (Type-it HRM PCR, Qiagen $\mathrm{GmbH}$, Hilden, Germany), $3.0 \mathrm{mmol} / \mathrm{l} \mathrm{MgCl}_{2}$ and $0.1 \mu \mathrm{mol} / / \mathrm{l}$ of each primer (forward: $5^{\prime}$-GTGGTGGAGGGAAGATGTTA-3' and reverse: 5'-TGCTTCGTGTTCATATGTTCG-3'). The HRM-PCR cycling and melting conditions were as follows: $95^{\circ} \mathrm{C}, 300 \mathrm{~s}$; 40 cycles of $\left(95^{\circ} \mathrm{C}, 10 \mathrm{~s} ; 55^{\circ} \mathrm{C}\right.$ fluorescence reading, $\left.10 \mathrm{~s} ; 72^{\circ} \mathrm{C}, 15 \mathrm{~s}\right)$. The HRM analysis was performed from $65^{\circ} \mathrm{C}$ to $95^{\circ} \mathrm{C}$ with gradual temperature increments of $0.1^{\circ} \mathrm{C} / \mathrm{s}$. Reactions were processed using the CFX96 real-time PCR system (Bio-Rad, Hercules, USA). All of the samples were tested in duplicate and control samples were included in each run.

\section{Results}

The c.5932G $>$ T, c.6095G >A, c.7630-2A $>C$ mutations in the 42, 43 and 54 exons of the ATM gene were analyzed in patients diagnosed with tobacco-related cancers $(n=783)$ and in healthy controls $(n=464)$.

The c.7630-2A>C mutation was identified only in 1 patient and in none of the control samples, while the c.5932G>T mutation was found neither in patients with tobacco-related cancers nor in controls. The c.6095G >A mutation was detected in one control sample.

The results of the RFLP, HRMA and sequencing analyses performed on the relevant samples are presented in Figure 1.

\section{Discussion}

Environmental factors such as cigarettes, alcohol, or environmental pollutants can be the cause of lesions in the DNA sequence [12]. However, in human cells several repair systems protect the genome and the DNA damage is corrected using various mechanisms, such as DSB repair, nucleotide excision repair (NER), mismatch repair (MMR), or base excision repair (BER) [13]. However, mutations in the DNA repair genes impair the DNA repair system and as a result can increase the risk of cancer development.

Several genes involved in the DNA repair process have been studied in a wide range of cancers in Polish patients. So far, only a few genes involved in BER (MPG gene) and NER (ERCC4 gene) as well as DSB (XRCC1, RAD51, NBN genes) repair systems have been examined among Polish patients. Chorazy el al. detected a few genetic variants in genes involved in repair of alkylated purines in the coding sequence of the MPG gene and in the enhancer of the MGMT gene in 33 nonsmall-cell lung cancer patients [14]. Szyfter et al. reported a statistically significant difference in frequency of genotypes 35931AC and 22541CA in $X P D$ between controls and primary head and neck cancer subjects $(\mathrm{OR}=0.49 ; 95 \% \mathrm{Cl}=0.29-0.85$; $p=0.010)$ and primary cancer subjects com pared with multiple cancer patients $(O R=2.30$ $95 \% \mathrm{Cl}=1.21-4.37 ; p=0.009$ ) [15]. On the other hand, it was found that polymorphisms in other repair genes such as XRCC 1 and ERCC4 are not associated with smoking and drinking in larynx cancer patients [16]. Morawiec-Szpondera et al. suggested that the rs1801320 variant (substitution of $G$ to $C$ at position 135 in $5^{\prime}$ untranslated region) of the RAD51 gene may be associated with smoking- and drinking-related larynx cancer in Poland [17]. We found that a mutation in a gene involved in DSB repair, p.I171V of the NBN gene, is associated with laryngeal cancer $(O R=11.70)$ and multiple primary tumors $(O R=28.35)$ and we also observed a significantly increased risk of lung cancer for the p.I171V variant $(\mathrm{OR}=7.76)[7,8]$.

In the Polish population the contribution of mutations in the ATM gene to head and neck and lung cancer development remains unexplored. To our knowledge, this is the first report in which mutations in the ATM gene were analyzed in parallel among three groups of tobacco-related cancer patients. This approach provided an assessment of the potential role of the ATM gene in cancer development in the Polish population.

Among Polish ataxia-telangiectasia patients a few recurring mutations were found in the ATM gene $[18,19]$. Three of the mutations $-c .6095 \mathrm{G}>\mathrm{A}$, c.7630-2A>C and c.5932G $>$ T - were the most frequent. Among 51 AT families, studied in the Institute of Human Genetics Polish Academy of Sciences, these mutations account for $35 \%$ of all detected ATM mutations. The mutation at position 
A

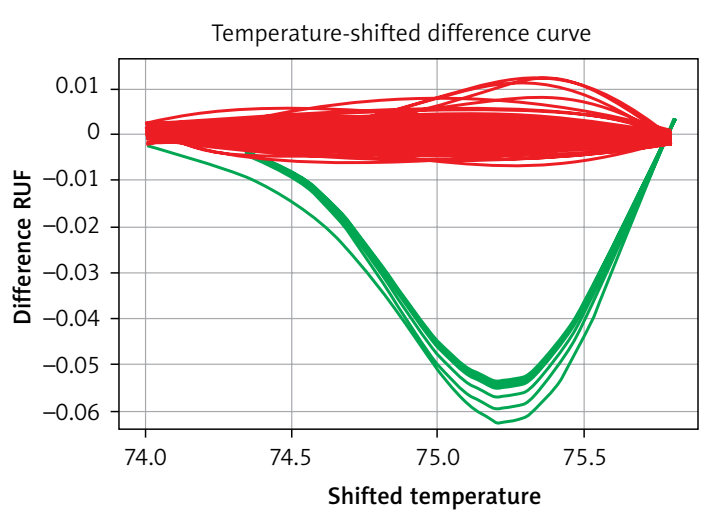

B

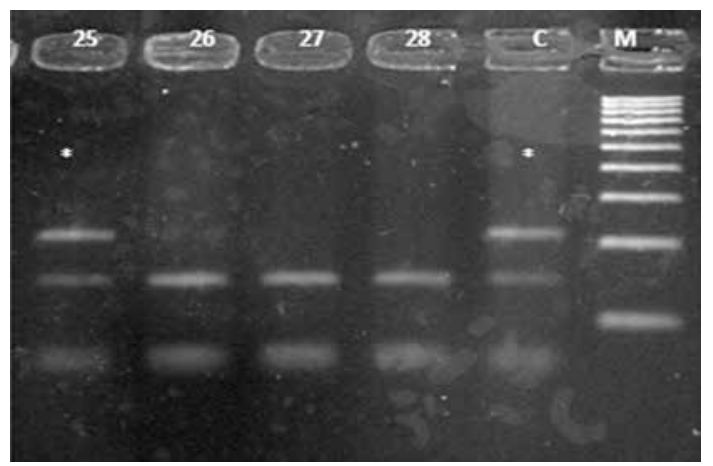

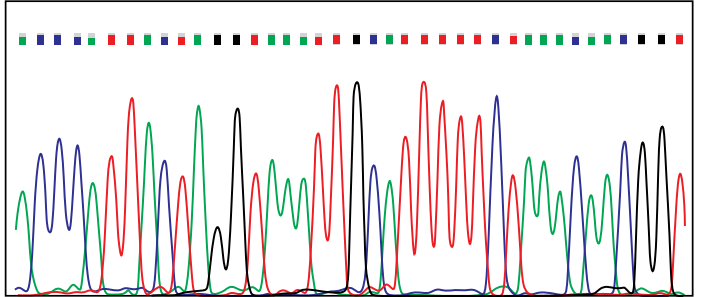
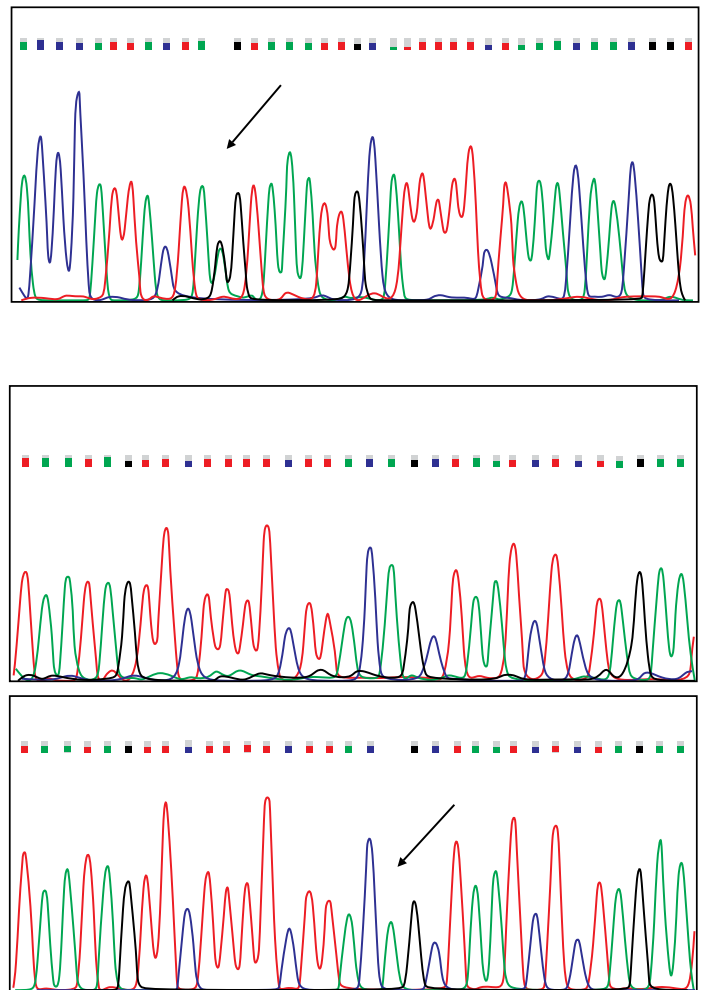

Figure 1. A - High resolution melting analysis and sequencing corresponding to the $c .6095 \mathrm{G}>\mathrm{A}$ variant. Red curves indicate GG genotype, green curves show heterozygous state (GA). In DNA sequencing plots variants are indicated by an arrow. B - Restriction fragment length polymorphism analysis and sequencing of the c.7630-2A>C variant. Stars indicate patients' mutant allele. $C$ indicates control sample. In DNA sequencing plots variants are indicated by an arrow

5932 changes a GAA codon into a UAA, resulting in the premature termination codon and protein truncation. The second mutation at position 6095 is a substitution of the last nucleotide of exon 43 and changes guanine to adenine. This mutation results in the deletion of exon 43 , caused by defective splicing. The third mutation, c.7630-2A $>C$, alters the splice-acceptor site from exon 54 and results in a deletion of exon 54 . All three mutations impair the kinase catalytic domain.

Several studies have indicated an association between the Polish recurring mutations and cancer development in the Polish population. Bogdanova et al. showed that c.5932G>T mutation is a predisposing breast cancer susceptibility variant in populations of Belarus, Russia, Ukraine and Poland [20]. A mutation at position 6095 was found in families with hereditary breast and ovarian cancer [21]. Moreover, c.6095G>A mutation was identified in patients with hereditary pancreatic cancer [3].
In addition, epidemiological studies confirmed the association between various ATM polymorphisms and different cancer risks, including lung cancer and head and neck cancer. Lo et al. found that nine ATM single nucleotide polymorphisms (rs189037, rs228597, rs228592, rs664677, rs609261, rs599558, rs609429, rs227062, and rs664982) increase the risk for lung cancer among never smokers [22]. A meta- analysis confirmed that ATM rs189037 polymorphism is associated with risk of head and neck cancer and lung cancer [23].

In the present study, no carriers of c.5932G $>T$, c.6095G $>$ A ATM mutations were found. Only one out of 783 patients with c.7630-2A>C mutation was detected (Figure 1). In the control group none of the C.5932G>T, c.7630-2A>C mutations were detected, but the c.6095G >A mutation was identified once (Figure 1).

Mutation c.7630-2A>C in exon 54 was identified in a female patient with lung cancer. In this 
case, tumor was diagnosed at age of 69 and was classified as adenocarcinoma. The pathologic stage of the tumor was defined as low grade (TNM classification: T1b, NO, M0). The patient has smoked for nearly 49 years (one pack of cigarettes per day). No other concomitant disease was diagnosed. Moreover, other cancers were observed among relatives. The woman's sister had ovarian cancer, and prostate cancer was diagnosed in the patient's father. The observed familial aggregation of cancer diseases suggests a common underlying genetic determinant of susceptibility to these diseases.

Based on this research, it can be concluded that studied mutations may have no influence in occurrence of head and neck cancer and lung cancer in Polish patients. There are some limitations to the current study that need to be addressed. The number of patients is limited, but the groups were very accurately selected and the history of the diseases and also exposure to tobacco and other noxious substances were collected in detail. It is possible that the association between ATM mutations and risk of head and neck cancer and lung cancer may be left out in a relatively small group of cases. The lack of evidence of the common ATM gene mutations in this preliminary study should be verified in replication studies. Depending on genetic heterogeneity of various cancers and population diversity, prospective studies with larger groups of patients may reveal the possible impact of the ATM gene in cancer occurrence.

In summary, it is known that genes which are not directly associated with cancer could modify cancer risk together or in combination with other factors. Taking this fact into consideration, patients with family history of multiple cancers should be especially screened for detection of ATM mutation carriers or low/moderate penetrance susceptibility allele variants in other genes. These ATM and other genes molecular variants may be interdependent predictors of phenotype in cancer diseases and should be considered in future studies concerning the development of gene-based prognostic scores for multiple cancers.

In conclusion, our study suggests that c.6095G $>A$, c.7630-2A>C, c.5932G>T ATM mutations have no influence on the occurrence of head and neck cancer and lung cancer in Polish patients.

\section{Acknowledgments}

Ministry of Science and Higher Education, Poland, grant no. NN401098240 and the National Science Centre, Poland, grant no. 2016/21/D/NZ5/00072.

\section{Conflict of interest}

The authors declare no conflict of interest.

\section{References}

1. Thompson D, Duedal S, Kirner J, et al. Cancer risks and mortality in heterozygous ATM mutation carriers. J Natl Cancer Inst 2005; 97: 813-22.

2. Helgason H, Rafnar T, Olafsdottir HS, et al. Loss-of-function variants in ATM confer risk of gastric cancer. Nat Genet 2015; 47: 906-10.

3. Roberts NJ, Jiao Y, Yu J, et al. ATM mutations in patients with hereditary pancreatic cancer. Cancer Discov 2012; 2: 41-6.

4. Bakker JL, de Winter JP. A role for ATM in hereditary pancreatic cancer. Cancer Discov 2012; 2: 14-5.

5. Leongamornlert D, Saunders E, Dadaev T, et al. Frequent germline deleterious mutations in DNA repair genes in familial prostate cancer cases are associated with advanced disease. Br J Cancer 2014; 110: 1663-72.

6. Zhao H, Albino AP, Jorgensen E, Traganos F, Darzynkiewicz Z. DNA damage response induced by tobacco smoke in normal human bronchial epithelial and A549 pulmonary adenocarcinoma cells assessed by laser scanning cytometry. Cytometry A 2009; 75: 840-7.

7. Ziolkowska I, Mosor M, Wierzbicka M, Rydzanicz M, Pernak-Schwarz M, Nowak J. Increased risk of larynx cancer in heterozygous carriers of the I171V mutation of the NBS1 gene. Cancer Sci 2007; 98: 1701-5.

8. Kaluzna EM, Rembowska J, Ziolkowska-Suchanek I, et al. Heterozygous p.I171V mutation of the NBN gene as a risk factor for lung cancer development. Oncol Lett 2015; 10: 3300-4.

9. Ziolkowska-Suchanek I, Mosor M, Wierzbicka M, Fichna M, Rydzanicz M, Nowak J. Association of polymorphisms and haplotypes of the NBN gene with laryngeal cancer and multiple primary tumors of the head and neck. Head Neck 2012; 34: 376-83.

10. Ziolkowska-Suchanek I, Mosor M, Gabryel P, et al. Susceptibility loci in lung cancer and COPD: association of IREB2 and FAM13A with pulmonary diseases. Sci Rep 2015; 5: 13502.

11. Ziolkowska-Suchanek I, Mosor M, Wierzbicka M, Rydzanicz M, Baranowska M, Nowak J. The MRN protein complex genes: MRE11 and RAD50 and susceptibility to head and neck cancers. Mol Cancer 2013; 12: 113.

12. Alexandrov LB, Ju YS, Haase K, et al. Mutational signatures associated with tobacco smoking in human cancer. Science 2016; 354: 618-22.

13. Chatterjee N, Walker GC. Mechanisms of DNA damage, repair, and mutagenesis. Environ Mol Mutagen 2017; 58: 235-63.

14. Rusin M, Samojedny A, Harris CC, Chorazy M. Novel genetic polymorphisms in DNA repair genes: O(6)-methylguanine-DNA methyltransferase (MGMT) and N-methylpurine-DNA glycosylase (MPG) in lung cancer patients from Poland. Hum Mutat 1999; 14: 269-70.

15. Szyfter K, Kujawski M, Gajęcka M, Rydzanicz M, Wierzbicka M. Relevance of DNA repair genes polymorphisms in an individual risk of multiple head and neck cancer. A preliminary report. Współcz Onkolol 2003; 7: 248-253.

16. Krupa R, Kasznicki J, Gajecka M, et al. Polymorphisms of the DNA repair genes XRCC1 and ERCC4 are not associated with smoking- and drinking-dependent larynx cancer in a Polish population. Exp Oncol 2011; 33: 55-6.

17. Romanowicz-Makowska H, Smolarz B, Gajecka M, et al. Polymorphism of the DNA repair genes RAD51 and XRCC2 in smoking- and drinking-related laryngeal cancer in a Polish population. Arch Med Sci 2012; 8: 1065-75. 
18. Podralska MJ, Stembalska A, Slezak R, et al. Ten new ATM alterations in Polish patients with ataxia-telangiectasia. Mol Genet Genomic Med 2014; 2: 504-11.

19. Mitui M, Bernatowska E, Pietrucha B, et al. ATM gene founder haplotypes and associated mutations in Polish families with ataxia-telangiectasia. Ann Hum Genet 2005; 69: 657-64.

20. Bogdanova N, Cybulski C, Bermisheva M, et al. A nonsense mutation (E1978X) in the ATM gene is associated with breast cancer. Breast Cancer Res Treat 2009; 118: 207-11.

21. Thorstenson YR, Roxas A, Kroiss R, et al. Contributions of ATM mutations to familial breast and ovarian cancer. Cancer Res 2003; 63: 3325-33.

22. Lo YL, Hsiao CF, Jou YS, et al. ATM polymorphisms and risk of lung cancer among never smokers. Lung Cancer 2010; 69: 148-54.

23. Bhowmik A, Nath S, Das S, Ghosh SK, Choudhury Y. ATM rs189037 (G > A) polymorphism and risk of lung cancer and head and neck cancer: a meta-analysis. Meta Gene 2015; 6: 42-8. 